\title{
Binary words containing infinitely many overlaps
}

\author{
James Currie \\ Department of Mathematics \\ University of Winnipeg \\ Winnipeg, Manitoba R3B 2E9 (Canada) \\ j.currie@uwinnipeg.ca \\ Narad Rampersad, Jeffrey Shallit \\ School of Computer Science \\ University of Waterloo \\ Waterloo, Ontario N2L 3G1 (Canada) \\ nrampersad@math. uwaterloo.ca \\ shallit@graceland.math. uwaterloo.ca
}

Submitted: Nov 16, 2005; Accepted: Sep 15, 2006; Published: Sep 22, 2006

Mathematics Subject Classifications: 68R15

\begin{abstract}
We characterize the squares occurring in infinite overlap-free binary words and construct various $\alpha$ power-free binary words containing infinitely many overlaps.
\end{abstract}

\section{Introduction}

If $\alpha$ is a rational number, a word $w$ is an $\alpha$ power if there exists words $x$ and $x^{\prime}$, with $x^{\prime}$ a prefix of $x$, such that $w=x^{n} x^{\prime}$ and $\alpha=n+\left|x^{\prime}\right| /|x|$. We refer to $|x|$ as a period of $w$. An $\alpha^{+}$power is a word that is a $\beta$ power for some $\beta>\alpha$. A word is $\alpha$ power-free (resp. $\alpha^{+}$power-free) if none of its subwords is an $\alpha$ power (resp. $\alpha^{+}$power). A 2 power is called a square; a $2^{+}$power is called an overlap.

Thue [18] constructed an infinite overlap-free binary word; however, Dekking [8] showed that any such infinite word must contain arbitrarily large squares. Shelton and Soni [17] characterized the overlap-free squares, but it is not hard to show that there are some overlap-free squares, such as 00110011, that cannot occur in an infinite overlap-free binary word. In this paper, we characterize those overlap-free squares that do occur in infinite overlap-free binary words.

Shur [16] considered the bi-infinite overlap-free and 7/3 power-free binary words and showed that these classes of words were identical. There have been several subsequent papers $[1,10,11,14]$ that have shown various similarities between the classes of overlap-free binary words and $7 / 3$ power-free binary words. Here we contrast the two classes of words 
by showing that there exist one-sided infinite $7 / 3$ power-free binary words containing infinitely many overlaps. More generally, we show that for any real number $\alpha>2$ there exists a real number $\beta$ arbitrarily close to $\alpha$ such that there exists an infinite $\beta^{+}$power-free binary word containing infinitely many $\beta$ powers.

All binary words considered in the sequel will be over the alphabet $\{0,1\}$. We therefore use the notation $\bar{w}$ to denote the binary complement of $w$; that is, the word obtained from $w$ by replacing 0 with 1 and 1 with 0 .

\section{Properties of the Thue-Morse morphism}

In this section we present some useful properties of the Thue-Morse morphism; i.e., the morphism $\mu$ defined by $\mu(0)=01$ and $\mu(1)=10$. It is well-known $[12,18]$ that the Thue-Morse word

$$
\mathbf{t}=\mu^{\omega}(0)=0110100110010110 \cdots
$$

is overlap-free.

The following property of $\mu$ is easy to verify.

Lemma 1. Let $x$ and $y$ be binary words. Then $x$ is a prefix (resp. suffix) of $y$ if and only if $\mu(x)$ is a prefix (resp. suffix) of $\mu(y)$.

Brandenburg [6] proved the following useful theorem, which was independently rediscovered by Shur [16].

Theorem 2 (Brandenburg; Shur). Let $w$ be a binary word and let $\alpha>2$ be a real number. Then $w$ is a power-free if and only if $\mu(w)$ is $\alpha$ power-free.

The following sharper version of one direction of this theorem (implicit in [10]) is also useful.

Theorem 3. Suppose $\mu(w)$ contains a subword $u$ of period $p$, with $|u| / p>2$. Then $w$ contains a subword $v$ of length $\lceil|u| / 2\rceil$ and period $p / 2$.

Karhumäki and Shallit [10] gave the following generalization of the factorization theorem of Restivo and Salemi [15]. The extension to infinite words is clear.

Theorem 4 (Karhumäki and Shallit). Let $x \in\{0,1\}^{*}$ be $\alpha$ power-free, $2<\alpha \leq 7 / 3$. Then there exist $u, v \in\{\epsilon, 0,1,00,11\}$ and an $\alpha$ power-free $y \in\{0,1\}^{*}$ such that $x=$ $u \mu(y) v$.

\section{$3 \quad$ Overlap-free squares}

Let

$$
A=\{00,11,010010,101101\}
$$


and let

$$
\mathcal{A}=\bigcup_{k \geq 0} \mu^{k}(A)
$$

Pansiot [13] and Brlek [7] gave the following characterization of the squares in $\mathbf{t}$.

Theorem 5 (Pansiot; Brlek). The set of squares in $\mathbf{t}$ is exactly the set $\mathcal{A}$.

We can use this result to prove the following.

Proposition 6. For any position $i$, there is at most one square in $\mathbf{t}$ beginning at position $i$.

Proof. Suppose to the contrary that there exist distinct squares $x$ and $y$ that begin at position $i$. Without loss of generality, suppose that $x$ and $y$ begin with 0 . Then by Theorem 5, $x=\mu^{p}(u)$ and $y=\mu^{q}(v)$, for some $p, q$ and $u, v \in\{00,010010\}$. Suppose $p \leq q$ and let $w=\mu^{q-p}(v)$. By Lemma 1, either $u$ is a proper prefix of $w$ or $w$ is a proper prefix of $u$, neither of which is possible for any choice of $u, v \in\{00,010010\}$.

The set $\mathcal{A}$ does not contain all possible overlap-free squares. Shelton and Soni [17] characterized the overlap-free squares (the result is also attributed to Thue in [4]).

Theorem 7 (Shelton and Soni). The overlap-free binary squares are the conjugates of the words in $\mathcal{A}$.

Some overlap-free squares cannot occur in any infinite overlap-free binary word, as the following lemma shows.

Lemma 8. Let $x=\mu^{k}(z)$ for some $k \geq 0$ and $z \in\{011011,100100\}$. Then $x$ a contains an overlap for all $a \in\{0,1\}$.

Proof. It is easy to see that $x=u v v u v v$ for some $u, v \in\{0,1\}^{*}$, where $u$ and $v$ begin with different letters. Thus one of uvvuvva or vva is an overlap.

We can characterize the squares that can occur in an infinite overlap-free binary word. Let

$$
B=\{001001,110110\}
$$

and let

$$
\mathcal{B}=\bigcup_{k \geq 0} \mu^{k}(B)
$$

Theorem 9. The set of squares that can occur in an infinite overlap-free binary word is $\mathcal{A} \cup \mathcal{B}$. Furthermore, if $\mathbf{w}$ is an infinite overlap-free binary word containing a subword $x \in \mathcal{B}$, then $\mathbf{w}$ begins with $x$ and there are no other occurrences of $x$ in $\mathbf{w}$. 
Proof. Let $\mathbf{w}$ be an infinite overlap-free binary word beginning with a square $y y \notin \mathcal{A} \cup \mathcal{B}$. Suppose further that $y y$ is a smallest such square that can be extended to an infinite overlap-free word. If $|y| \leq 3$, then $y y \notin \mathcal{A} \cup \mathcal{B}$ is one of 011011 or 100100 , neither of which can be extended to an infinite overlap-free word by Lemma 8.

We assume then that $|y|>3$. Since, by Theorem $7, y y$ is a conjugate of a word in $\mathcal{A}$, we have two cases.

Case 1: $y y=\mu(z z)$ for some $z \in\{0,1\}^{*}$. By Theorem $4, \mathbf{w}=\mu\left(z z \mathbf{w}^{\prime}\right)$ for some infinite $\mathbf{w}^{\prime}$, where $z z \mathbf{w}^{\prime}$ is overlap-free. Thus $z z$ is a smaller square not in $\mathcal{A} \cup \mathcal{B}$ that can be extended to an infinite overlap-free word, contrary to our assumption.

Case 2: $y y=a \mu\left(z z^{\prime}\right) \bar{a}$ for some $a \in\{0,1\}$ and $z, z^{\prime} \in\{0,1\}^{*}$. By Theorem $4, y y$ is followed by $a$ in $\mathbf{w}$, and so yya is an overlap, contrary to our assumption.

Since both cases lead to a contradiction, our assumption that yy $\notin \mathcal{A} \cup \mathcal{B}$ must be false.

To see that each word in $\mathcal{A} \cup \mathcal{B}$ does occur in some infinite overlap-free binary word, note that Allouche, Currie, and Shallit [2] have shown that the word $\mathbf{s}=001001 \overline{\mathbf{t}}$ is overlap-free. Now consider the words $\mu^{k}(\mathbf{s})$ and $\mu^{k}(\overline{\mathbf{s}})$, which are overlap-free for all $k \geq 0$.

Finally, to see that any occurrence of $x \in \mathcal{B}$ in $\mathbf{w}$ must occur at the beginning of $\mathbf{w}$, we note that by an argument similar to that used in Lemma 8, ax contains an overlap for all $a \in\{0,1\}$, and so $x$ occurs at the beginning of $\mathbf{w}$.

\section{Words containing infinitely many overlaps}

In this section we construct various infinite $\alpha$ power-free binary words containing infinitely many overlaps. We begin by considering the infinite $7 / 3$ power-free binary words.

Proposition 10. For all $p \geq 1$, an infinite $7 / 3$ power-free word contains only finitely many occurrences of overlaps with period $p$.

Proof. Let $\mathbf{x}$ be an infinite $7 / 3$ power-free word containing infinitely many overlaps with period $p$. Let $k \geq 0$ be the smallest integer satisfying $p \leq 3 \cdot 2^{k}$. Suppose $\mathbf{x}$ contains an overlap $w$ with period $p$ starting in a position $\geq 2^{k+1}$. Then by Theorem 4 , we can write

$$
\mathbf{x}=u_{1} \mu\left(u_{2}\right) \cdots \mu^{k-1}\left(u_{k}\right) \mu^{k}(\mathbf{y}),
$$

where each $u_{i} \in\{\epsilon, 0,1,00,11\}$. The overlap $w$ occurs as a subword of $\mu^{k}(\mathbf{y})$. By Lemma 3 , $\mathbf{y}$ contains an overlap with period $p / 2^{k} \leq 3$. But any overlap with period $\leq 3$ contains a $7 / 3$ power. Thus, $\mathbf{x}$ contains a $7 / 3$ power, a contradiction.

The following theorem provides a striking contrast to Shur's result [16] that the biinfinite $7 / 3$ power-free words are overlap-free.

Theorem 11. There exists a 7/3 power-free binary word containing infinitely many overlaps. 
Proof. We define the following sequence of words: $A_{0}=00$ and $A_{n+1}=0 \mu^{2}\left(A_{n}\right), n \geq 0$. The first few terms in this sequence are

$$
\begin{aligned}
& A_{0}=00 \\
& A_{1}=001100110 \\
& A_{2}=0011001101001100101100110100110010110
\end{aligned}
$$

We first show that in the limit as $n \rightarrow \infty$, this sequence converges to an infinite word a. It suffices to show that for all $n, A_{n}$ is a prefix of $A_{n+1}$. We proceed by induction on n. Certainly, $A_{0}=00$ is a prefix of $A_{1}=0 \mu^{2}(00)=001100110$. Now $A_{n}=0 \mu^{2}\left(A_{n-1}\right)$, $A_{n+1}=0 \mu^{2}\left(A_{n}\right)$, and by induction, $A_{n-1}$ is a prefix of $A_{n}$. Applying Lemma 1 , we see that $A_{n}$ is a prefix of $A_{n+1}$, as required.

Note that for all $n, A_{n+1}$ contains $\mu^{2 n}\left(A_{1}\right)$ as a subword. Since $A_{1}$ is an overlap with period $4, \mu^{2 n}\left(A_{1}\right)$ contains $2^{2 n}$ overlaps with period $2^{2 n+2}$. Thus, a contains infinitely many overlaps.

We must show that a does not contain a $7 / 3$ power. It suffices to show that $A_{n}$ does not contain a $7 / 3$ power for all $n \geq 0$. Again, we proceed by induction on $n$. Clearly, $A_{0}=00$ does not contain a $7 / 3$ power. Consider $A_{n+1}=0 \mu^{2}\left(A_{n}\right)$. By induction, $A_{n}$ is $7 / 3$ power-free, and by Theorem 2 , so is $\mu^{2}\left(A_{n}\right)$. Thus, if $A_{n+1}$ contains a $7 / 3$ power, such a $7 / 3$ power must occur as a prefix of $A_{n+1}$. Note that $A_{n+1}$ begins with 00110011 . The word 00110011 cannot occur anywhere else in $A_{n+1}$, as that would imply that $A_{n+1}$ contained a cube 000 or 111 , or the $5 / 2$ power 1001100110 . If $A_{n+1}$ were to begin with a $7 / 3$ power with period $\geq 8$, it would contain two occurrences of 00110011 , contradicting our earlier observation. We conclude that the period of any such $7 / 3$ power is less than 8 . Checking that no such $7 / 3$ power exists is now a finite check and is left to the reader.

In fact, we can prove the following stronger statement.

Theorem 12. There exist uncountably many $7 / 3$ power-free binary words containing infinitely many overlaps.

Proof. For a finite binary sequence $b$, we define an operator $g_{b}$ on binary words recursively by

$$
\begin{aligned}
g_{\epsilon}(w) & =w \\
g_{0 b}(w) & =\mu^{2}\left(g_{b}(w)\right) \\
g_{1 b}(w) & =0 \mu^{2}\left(g_{b}(w)\right) .
\end{aligned}
$$

Note that $g_{b}(0)$ always starts with a 0 , so that for any finite binary words $p$ and $b, g_{p}(0)$ is always a prefix of $g_{p b}(0)$. Since $g_{0}(0)$ is not a prefix of $g_{1}(0), g_{p 0}(0)$ is not a prefix of $g_{p 1}(0)$ for any $p$, so that distinct $b$ give distinct words. Given an infinite binary sequence $\mathbf{b}=b_{1} b_{2} b_{3} \cdots$ where the $b_{i} \in\{0,1\}$, define an infinite binary sequence $w_{\mathbf{b}}$ to be the limit of

$$
g_{\epsilon}(00), g_{b_{1}}(00), g_{b_{1} b_{2}}(00), g_{b_{1} b_{2} b_{3}}(00), \ldots
$$


By an earlier argument, each $w_{\mathbf{b}}$ is $7 / 3$ power-free. Since $g_{1}(00)=001100110$ is an overlap, $g_{b 1}(00)=g_{b}(001100110)$ ends with an overlap for any finite word $b$. Thus, each 1 in $\mathbf{b}$ introduces an overlap in $w_{\mathbf{b}}$. Since uncountably many binary sequences contain infinitely many 1 's, uncountably many of the $w_{\mathbf{b}}$ are $7 / 3$ power-free words containing infinitely many overlaps.

Next, we show that the sequence a constructed in the proof of Theorem 11 is an automatic sequence (in the sense of [3]).

Proposition 13. The sequence a is 4-automatic.

Proof. We show that $\mathbf{a}=g\left(h^{\omega}(0)\right)$, where $h$ and $g$ are the morphisms defined by

$$
\begin{aligned}
& h(0)=0134 \\
& h(1)=2134 \\
& h(2)=3234 \\
& h(3)=2321 \\
& h(4)=3421
\end{aligned}
$$

and

$$
\begin{aligned}
& g(0)=0 \\
& g(1)=0 \\
& g(2)=0 \\
& g(3)=1 \\
& g(4)=1 .
\end{aligned}
$$

We make some observations concerning 2-letter subwords: The sequence $h^{\omega}(0)$ clearly does not contain any of the words 11, 14, 22, 24, 31, 33, 41 or 44 . In fact, neither 12 nor 43 appears as a subword either: Words 12 and 43 do not appear internally in $h(i), 0 \leq i \leq 4$; therefore, if 43 appears in $h^{n}(0)$, it must 'cross the boundary' in one of $h(12), h(14), h(22)$ or $h(24)$. Since 14, 22 and 24 do not appear in $h^{\omega}(0)$, word 43 can only appear in $h^{n}(0)$ as a descendant of a subword 12 in $h^{n-1}(0)$. However, the situation is symmetrical; word 12 can only appear in $h^{n}(0)$ as a descendant of a subword 43 in $h^{n-1}(0)$. By induction, neither 43 nor 12 ever appears.

The point of the previous paragraph is that

$$
\begin{aligned}
& h(0) \text { always occurs in the context } h(0) 2 \\
& h(1) \text { always occurs in the context } h(1) 2 \\
& h(2) \text { always occurs in the context } h(2) 2 \\
& h(3) \text { always occurs in the context } h(3) 3 \\
& h(4) \text { always occurs in the context } h(4) 3
\end{aligned}
$$

The word $h^{\omega}(0)$ can thus be parsed in terms of a new morphism $f$ :

$$
\begin{aligned}
& f(0)=1342 \\
& f(1)=1342 \\
& f(2)=2342 \\
& f(3)=3213 \\
& f(4)=4213 .
\end{aligned}
$$


The parsing in terms of $f$ works as follows: If we write $h^{\omega}(0)=0 w$, then $w=f(0 w)$. It is useful to rewrite this relation in terms of the finite words $h^{n}(0)$. For non-negative integer $n$ let $x_{n}$ be the unique letter such that $h^{n}(0) x_{n}$ is a prefix of $h^{\omega}(0)$. Thus $x_{0}=1$, $x_{1}=2$, etc. We then have

$$
h^{n}(0) x_{n}=0 f\left(h^{n-1}(0)\right), \quad n \geq 1 .
$$

Since for all $a \in\{0,1,2,3,4\}, g(f(a))=\mu^{2}(g(a))$, we have $g(f(u))=\mu^{2}(g(u))$ for all words $u$. Therefore, applying $g$ to (1)

$$
\begin{aligned}
g\left(h^{n}(0) x_{n}\right) & =g\left(0 f\left(h^{n-1}(0)\right)\right) \\
& =g(0) g\left(f\left(h^{n-1}(0)\right)\right) \\
& =0 \mu^{2}\left(g\left(h^{n-1}(0)\right)\right), \quad n \geq 1 .
\end{aligned}
$$

From this relation we show by induction that $A_{n}$ is the prefix of $g\left(h^{n+1}(0)\right)$ of length $\left(4^{n+1}+3 \cdot 4^{n}-1\right) / 3$. Certainly, $A_{0}=00$ is the prefix of length 2 of $g(h(0))=0011$. Consider $A_{n}=0 \mu^{2}\left(A_{n-1}\right)$. We can assume inductively that $A_{n-1}$ is the prefix of $g\left(h^{n}(0)\right)$ of length $\left(4^{n}+3 \cdot 4^{n-1}-1\right) / 3$. Writing $g\left(h^{n}(0)\right)=A_{n-1} z$ for some $z$, we have

$$
\begin{aligned}
g\left(h^{n+1}(0) x_{n+1}\right) & =0 \mu^{2}\left(g\left(h^{n}(0)\right)\right) \\
& =0 \mu^{2}\left(A_{n-1} z\right) \\
& =A_{n} \mu^{2}(z),
\end{aligned}
$$

for some $x_{n+1}$, whence $A_{n}$ is a prefix of $g\left(h^{n+1}(0)\right)$. Since $\left|A_{n}\right|=4\left|A_{n-1}\right|+1$, we have $\left|A_{n}\right|=\left(4^{n+1}+3 \cdot 4^{n}-1\right) / 3$, as required.

The result of Theorem 11 can be strengthened even further.

Theorem 14. For every real number $\alpha>2$ there exists a real number $\beta$ arbitrarily close to $\alpha$, such that there is an infinite $\beta^{+}$power-free binary word containing infinitely many $\beta$ powers.

Proof. Let $s \geq 3$ be a positive integer, and let $r=\lfloor\alpha+1\rfloor$. Let $t$ be the largest positive integer such that $r-t / 2^{s}>\alpha$, and such that the word obtained by removing a prefix of length $t$ from $\mu^{s}(0)$ begins with 00 . Let $\beta=r-t / 2^{s}$. Since $\alpha \geq r-1$, we have $t<2^{s}$. Also, $\mu^{3}(0)=01101001$ and $\mu^{3}(1)=10010110$ are of length 8 , and both contain 00 as a subword; it follows that $|\alpha-\beta| \leq 8 / 2^{s}$, so that by choosing large enough $s, \beta$ can be made arbitrarily close to $\alpha$.

We construct sequences of words $A_{n}, B_{n}$ and $C_{n}$. Define $C_{0}=00$. For each $n \geq 0$ :

1. Let $A_{n}=0^{r-2} C_{n}$.

2. Let $B_{n}=\mu^{s}\left(A_{n}\right)$.

3. Remove the first $t$ letters from $B_{n}$ to obtain a new word $C_{n+1}$ beginning with 00 . 
Since each $A_{n}$ begins with the $r$ power $0^{r}$, each $B_{n}=\mu^{s}\left(A_{n}\right)$ begins with an $r$ power of period $2^{s}$. Removing the first $t$ letters ensures that $C_{n+1}$ commences with an $\left(r 2^{s}-t\right) / 2^{s}$ power, viz., a $\beta$ power. The limit of the $C_{n}$ gives the desired infinite word. Let us check that this limit exists:

Let $w$ be the word consisting of the first $t$ letters of $\mu^{s}(0)$. Since all the $A_{n}$ commence with 0 by construction, all the $B_{n}$ commence with $\mu^{s}(0)$, and hence with $w$. This means that $B_{n}=w C_{n+1}$ for each $n$.

We show that $A_{n}$ is always a prefix of $A_{n+1}$ by induction. Certainly $A_{0}$ is a prefix of $A_{1}$. Assume that $A_{n-1}$ is a prefix of $A_{n}$. Since $A_{n}=0^{r-2} C_{n}$ and $A_{n+1}=0^{r-2} C_{n+1}, A_{n}$ is a prefix of $A_{n+1}$ if $C_{n}$ is a prefix of $C_{n+1}$. Since $B_{n-1}=w C_{n}$ and $B_{n}=w C_{n+1}, C_{n}$ is a prefix of $C_{n+1}$ if $B_{n-1}$ is a prefix of $B_{n}$. By Lemma $1, B_{n-1}$ is a prefix of $B_{n}$ if $A_{n-1}$ is a prefix of $A_{n}$, which is our inductive assumption. We conclude that $A_{n}$ is a prefix of $A_{n+1}$.

It follows that $C_{n}$ is a prefix of $C_{n+1}$ for $n \geq 0$, so that the limit of the $C_{n}$ exists. It will thus suffice to prove the following claim:

Claim: The $A_{n}, B_{n}$ and $C_{n}$ satisfy the following:

1. The word $C_{n}$ contains no $\beta^{+}$powers.

2. The only $\beta^{+}$power in $A_{n}$ is $0^{r}$.

3. Any $\beta^{+}$powers in $B_{n}$ appear only in the prefix $\mu^{s}\left(0^{r}\right)$.

Certainly $C_{0}$ contains no $\beta^{+}$powers, and since $\beta>r-1$, the only $\beta^{+}$power in $A_{0}$ is $0^{r}$. Suppose then that the claim holds for $A_{n}$ and $C_{n}$.

Now suppose that $B_{n}=\mu^{s}\left(0^{r-2}\right) \mu^{s}\left(C_{n}\right)$ contains a $\beta^{+}$power $u$ with period $p$. Since $C_{n}$ contains no $\beta^{+}$powers, Theorem 2 ensures that $\mu^{s}\left(C_{n}\right)$ contains no $\beta^{+}$powers. We can therefore write $B_{n}=x u y$ where $|x|<\left|\mu^{s}\left(0^{r-2}\right)\right|$. In other words, $u$ overlaps $\mu^{s}\left(0^{r-2}\right)$ from the right. By Theorem 3, the preimage of $B_{n}$ under $\mu$, i.e., $\mu^{s-1}\left(A_{n}\right)$, contains a $\beta^{+}$power of length at least $|u| / 2$ and period $p / 2$. In fact, iterating this argument, $A_{n}$ contains a $\beta^{+}$power of period $p / 2^{s}$ of length at least $|u| / 2^{s}$. Since the only $\beta^{+}$power in $A_{n}$ is $0^{r}$, with period 1 , we see that $p / 2^{s}=1$, whence $p=2^{s}$ and $|u| \leq r 2^{s}$.

Recall that $B_{n}$ has a prefix $\mu^{s}\left(0^{r}\right)$ which also has period $2^{s}$, and that this prefix is overlapped by $u$. It follows that all of $x u$ is a $\beta^{+}$power with period $p=2^{s}$. However, as just argued, this means that $|x u| \leq r 2^{s}=\left|\mu^{s}\left(0^{r}\right)\right|$, so that $u$ is contained in $\mu^{s}\left(0^{r}\right)$ and part 3 of our claim holds for $B_{n}$. We now show that parts 1 and 2 hold for $C_{n+1}$ and $A_{n+1}$ respectively, and the truth of our claim will follow by induction.

Part 1 follows immediately from part 3.

Now suppose that $A_{n+1}$ contains a $\beta^{+}$power $u$. Recall that $A_{n+1}=0^{r-2} C_{n+1}$, and $C_{n+1}$ begins with 00 , but contains no $\beta^{+}$powers. It follows that $u$ is not a subword of $C_{n+1}$. Therefore, 000 must be a prefix of $u$. If $u=0^{q}$ for some integer $q$, then $q \leq r$ by the construction of $A_{n+1}$, and

$$
r \geq q>\beta>\alpha>r-1 .
$$

This implies that $q=r$, and $u=0^{r}$, as claimed. If we cannot write $u=0^{q}$, then $|u|_{1} \geq 1$. Because $u$ is a $2^{+}$power, 000 must appear twice in $u$ with a 1 lying somewhere between the 
two appearances. This implies that 000 is a subword of $C_{n+1}$, and hence of $B_{n}=\mu^{s}\left(A_{n}\right)$. However, no word of the form $\mu(w)$ contains 000. This is a contradiction.

We conclude by presenting the following open problem.

Does there exist a characterization (in the sense of $[5,9]$ ) of the infinite $7 / 3$ power-free binary words?

\section{Acknowledgments}

Thanks to the referee for pointing out Brandenburg's proof of Theorem 2.

\section{References}

[1] A. Aberkane, J. Currie, "Attainable lengths for circular binary words avoiding $k$ powers", Bull. Belg. Math. Soc. Simon Stevin, 2004, to appear.

[2] J.-P. Allouche, J. Currie, J. Shallit, "Extremal infinite overlap-free words", Electron. J. Combin. 5 (1998), \#R27.

[3] J.-P. Allouche, J. Shallit, Automatic Sequences: Theory, Applications, Generalizations, Cambridge, 2003.

[4] J. Berstel, "Axel Thue's work on repetitions in words". In P. Leroux, C. Reutenauer, eds., Séries formelles et combinatoire algébrique, Publications du LaCIM, pp 65-80, UQAM, 1992.

[5] J. Berstel, "A rewriting of Fife's theorem about overlap-free words". In J. Karhumäki, H. Maurer, G. Rozenberg, eds., Results and Trends in Theoretical Computer Science, Vol. 812 of Lecture Notes in Computer Science, pp. 19-29, Springer-Verlag, 1994.

[6] F.-J. Brandenburg, "Uniformly growing $k$-th power-free homomorphisms", Theoret. Comput. Sci. 23 (1983), 69-82.

[7] S. Brlek, "Enumeration of factors in the Thue-Morse word", Discrete Appl. Math. 24 (1989), 83-96.

[8] F. M. Dekking, "On repetitions in binary sequences", J. Comb. Theory Ser. A 20 (1976), 292-299.

[9] E. Fife, "Binary sequences which contain no BBb", Trans. Amer. Math. Soc. 261 (1980), 115-136.

[10] J. Karhumäki, J. Shallit, "Polynomial versus exponential growth in repetition-free binary words", J. Combin. Theory Ser. A 104 (2004), 335-347. 
[11] R. Kolpakov, G. Kucherov, Y. Tarannikov, "On repetition-free binary words of minimal density", WORDS (Rouen, 1997), Theoret. Comput. Sci. 218 (1999), 161-175.

[12] M. Morse, G. Hedlund, "Unending chess, symbolic dynamics, and a problem in semigroups", Duke Math. J. 11 (1944), 1-7.

[13] J. J. Pansiot, "The Morse sequence and iterated morphisms", Inform. Process. Lett. 12 (1981), 68-70.

[14] N. Rampersad, "Words avoiding $\frac{7}{3}$-powers and the Thue-Morse morphism", Internat. J. Found. Comput. Sci. 16 (2005), 755-766.

[15] A. Restivo, S. Salemi, "Overlap free words on two symbols". In M. Nivat, D. Perrin, eds., Automata on Infinite Words, Vol. 192 of Lecture Notes in Computer Science, pp. 198-206, Springer-Verlag, 1984.

[16] A. M. Shur, "The structure of the set of cube-free $\mathbb{Z}$-words in a two-letter alphabet" (Russian), Izv. Ross. Akad. Nauk Ser. Mat. 64 (2000), 201-224. English translation in Izv. Math. 64 (2000), 847-871.

[17] R. Shelton, R. Soni, "Chains and fixing blocks in irreducible binary sequences", Discrete Math. 54 (1985), 93-99.

[18] A. Thue, "Über die gegenseitige Lage gleicher Teile gewisser Zeichenreihen", Kra. Vidensk. Selsk. Skrifter. I. Math. Nat. Kl. 1 (1912), 1-67. 